C. Huneke and B. Ulrich

Nagoya Math. J.

Vol. 109 (1988), 159-167

\title{
MINIMAL LINKAGE AND THE GORENSTEIN LOCUS OF AN IDEAL
}

\author{
CRAIG HUNEKE* AND BERND ULRICH*
}

\section{Introduction}

Let $I$ be a Cohen-Macaulay ideal of grade $g>0$ in a local Gorenstein ring $(R, m)$ with residue class field $k$. An $R$-ideal $J$ is said to be linked to $I$ with respect to the regular sequence $\underline{\alpha}=\alpha_{1}, \cdots, \alpha_{g} \subset I \cap J$ if $J=$ $(\underline{\alpha}): I$ and $I=(\underline{\alpha}): J([6])$. In this paper we are concerned with the following question: how big is $\operatorname{dim}_{k}((\underline{\alpha}, m J) / m J)$ ? Obviously this dimension is at most $g$, but it could be as small as 0 . If it is $g$ then the link from $J$ to $I$ is called a minimal link, which is in most respects the desired type of link. The only general result known in this direction is that if $I$ is Gorenstein, then $\operatorname{dim}_{k}((\underline{\alpha}, m J) / m J)=g$ unless both $I$ and $J$ are complete intersections (see [1], Proposition 5.2). We are able to generalize this fact to the case where $(R / I)_{p}$ is Gorenstein for all prime ideals $p$ in $R / I$ with $\operatorname{dim}(R / I)_{p} \leq 4$; however we have to assume that $I$ is generically a complete intersection ideal, and that $R$ is a complete intersection (Theorem 2.3). Without the assumption on $R$ we prove that if $I$ is generically a complete intersection, and if for a fixed integer $r$ the type of $(R / I)_{p}$ is at most $r$ for all prime ideals $p$ in $R / I$ with $\operatorname{dim}(R / I)_{p} \leq(r+1)^{2}$, then $\operatorname{dim}_{k}((\underline{\alpha}, m J / m J)) \geq g-r$ (Proposition 2.1). If $r=1$, i.e. if $R / I$ is Gorenstein in codimension 4, then this estimate shows the dimension is at least $g-1$. Theorem 2.3 can also be interpreted to yield a strong upper bound for the codimension of the non-Gorenstein-locus of certain perfect ideals: Let $R$ be a regular local ring. Let $I$ be an $R$-ideal which is generically a complete intersection, and assume that $I$ is in the even linkage class of a Gorenstein ideal (i.e., there exists a sequence of links $I \sim I_{1} \sim I_{2} \sim \cdots \sim I_{2 n}$ with $I_{2 n}$ a Gorenstein ideal); then $I$ is a Gorenstein ideal provided that $(R / I)_{p}$ is Gorenstein for all prime ideals $p$ of $R / I$ with $\operatorname{dim}(R / I)_{p} \leq 4$ (Corollary 3.1).

Received December 10, 1986.

* Partially supported by the NSF. 


\section{§1. General facts about linkage}

In this section, we fix the notations we will be using throughout the paper and review some definitions and results from [4].

Let $(R, m)$ be a local Noetherian ring, let $I$ be an $R$-ideal, and $M$ a finitely generated $R$-module. By $\nu(M)$ we denote the minimal number of generators of $M, \operatorname{ht}(I)$ is the height of $I$ and $r(R)=\operatorname{dim}_{R / n}\left(\operatorname{Ext}_{R}^{d}(R / m, R)\right)$ stands for the type of $R$ (if $R$ is Cohen-Macaulay of dimension $d$ ). We say that $I$ is Cohen-Macaulay or Gorenstein if the ring $R / I$ has any of these properties. The ideal $I$ is a complete intersection if $I$ is generated by a regular sequence, $I$ is called generically a complete intersection if $I$ is unmixed and $I_{p}$ is a complete intersection for all $p \in \operatorname{Ass}(R / I)$, and $I$ is an almost complete intersection if $\nu(I) \leq$ grade $(I)+1$. We say that $R$ is a complete intersection if $\hat{R}$ is a regular local ring modulo a complete intersection ideal. For an integer $k, R$ satisfies $\left(R_{k}\right)$ if $R_{p}$ is regular for all $p \in \operatorname{Spec}(R)$ with $\operatorname{dim} R_{p} \leq k, R$ is $\left(G_{k}\right)$ if $R_{p}$ is Gorenstein for all $p \in \operatorname{Spec}(R)$ with $\operatorname{dim} R_{p} \leq k$, and $I$ satisfies $\left(C I_{k}\right)$ if $I_{p}$ is a complete intersection for all $p \in \operatorname{Spec}(R / I)$ with $\operatorname{dim}(R / I)_{p} \leq k$. For a matrix $A$ with entries in $R, I_{t}(A)$ is the $R$-ideal generated by all $t \times t$ minors of $A$, and for a set of elements $\underline{f}=f_{1}, \cdots, f_{n} \subset R$ we will denote by $(\underline{f})$ the $R$-ideal generated by $f_{1}, \cdots, f_{n}$ whereas $(f)^{t}$ stands for the transpose of the matrix $\left(f_{1} \cdots f_{n}\right)$. If $X$ is a finite set of indeterminates we set $R(X)=R[X]_{m R[X]}$.

Definition $1.1([4])$. Let $(R, I)$ and $(S, J)$ be pairs of Noetherian local rings $R, S$, and ideals $I \subset R, J \subset S$.

a) $(S, J)$ is a deformation of $(R, I)$ (with respect to $\underline{a}$ ) if there is a sequence $\underline{a} \subset S$ which is regular on $S$ and $S / J$ such that $(S /(\underline{a}),(J, \underline{a}) /(\underline{a}))$ $=(R, I)$.

b) $(S, J)$ and $(R, I)$ are equivalent if there are finite sets of variables $X$ over $S$, and $Z$ over $R$, and an isomorphism $\varphi: S[X] \stackrel{\sim}{\longrightarrow} R[Z]$ such that $\varphi(J S[X])=I R[Z]$.

Definition 1.2 ([6]). Let $R$ be a local Cohen-Macaulay ring, and let $I$ and $J$ be two (proper) $R$-ideals, then $I$ and $J$ are said to be (algebraically) linked (with respect to $\underline{\alpha}$ ) (written $I \sim J$ ), if there exists a regular sequence $\underline{\alpha}=\alpha_{1}, \cdots, \alpha_{g} \subset I \cap J$ such that $J=(\underline{\alpha}): I$ and $I=(\underline{\alpha}): J$.

It is known that if $R$ is a local Gorenstein ring, $I$ an unmixed $R$ ideal of grade $g$, and $\underline{\alpha}=\alpha_{1}, \cdots, \alpha_{g} \subset I$ a regular sequence with $(\underline{\alpha}) \neq I$, then $J=(\underline{\alpha}): I$ is linked to $I$ ([6]). If moreover $I$ is Cohen-Macaulay, 
then $J$ is Cohen-Macaulay, and $J /(\underline{\alpha})$ is the canonical module of $R / I([6])$. Hence $\nu(J /(\underline{\alpha}))=r(R / I)$, and in particular, $\nu(J)=r(R / I)+g$ if and only if $\underline{\alpha}=\alpha_{1}, \cdots \alpha_{g}$ form part of a minimal generating set of $J$. In this case, we say that the link from $J$ to $I$ is minimal. Two $R$-ideals $I$ and $J$ are said to be in the same linkage class if there is a sequence of $n$ links $I=I_{0} \sim I_{1} \sim \cdots \sim I_{n}=J$. If in addition $n$ can be chosen to be even, then $I$ and $J$ are in the same even linkage class.

Definition 1.3 ([3], [4]). Let $R$ be a local Gorenstein ring, let $I$ be an unmixed $R$-ideal of grade $g$, fix a generating sequence $\underline{f}=f_{1}, \cdots, f_{n}$ of $I$, let $X=\left(X_{i j}\right)$ be a generic $g \times n$ matrix, let $S=R[X],\left(\begin{array}{c}\alpha_{1} \\ \vdots \\ \alpha_{g}\end{array}\right)=X\left(\begin{array}{c}f_{1} \\ \vdots \\ f_{n}\end{array}\right)$. Then $\underline{\alpha}=\alpha_{1}, \cdots, \alpha_{g} \subset I S$ is an $S$-regular sequence, and we call $L_{1}(\underline{f})=$ $(\underline{\alpha}) S: I S \subset S$ a first generic link of $I$.

In [4], 2.11, it is shown that up to equivalence in the sense of Definition $1.1 \mathrm{~b}$, the pair $\left(S, L_{1}(f)\right)$ only depends on $I$, but not on the chosen generating sequence $\underline{f}$. Hence we write $L_{1}(I)$ instead of $L_{1}(f)$. In [4], 2.13, we also remarked that if $L_{1}(I) \subset R[X]$ is a first generic link of $I$, and $p \in \operatorname{Spec}(R), I \subset p$, then $L_{1}(I) R_{p}[X]$ is a first generic link of $I_{p}$. We will use the following property of generic links.

Proposition 1.4 ([4]). Let $(R, m)$ be a local Gorenstein ring, let $I$ be a Cohen-Macaulay $R$-ideal, and let $J$ be linked to $I$ with respect to the regular sequence $\underline{\alpha}=\alpha_{1}, \cdots, \alpha_{g}$. Fix a generating sequence $\underline{f}=f_{1}, \cdots, f_{n}$ of $I$ and $a g \times n$ matrix $C=\left(C_{i j}\right)$ with entries in $R$ such that $(\underline{\alpha})^{t}=C(\underline{f})^{t}$. Let $L_{1}(f) \subset R[X]$ be a first generic link as defined in 1.3 , and consider $p=$ $\left(m, X_{i j}-C_{i j}\right) R[X] \in \operatorname{Spec}(R[X])$.

Then $\left(R[X]_{p}, L_{1}(\underline{f}) R[X]_{p}\right)$ is a deformation of $(R, J)$.

\section{§2. Minimal linkage}

For the proof of the main result (Theorem 2.3) we need two propositions which might also be of independent interest.

Proposition 2.1. Let $(R, m)$ be a local Gorenstein ring with residue class field $k$, let $I$ be a Cohen-Macaulay $R$-ideal of grade $g$ which is generically a complete intersection, and assume that there is an integer $r$ such that $r\left((R / I)_{p}\right) \leq r$ for all $p \in \operatorname{Spec}(R / I)$ with $\operatorname{dim}(R / I)_{p} \leq(r+1)^{2}$. Let $J$ be an $R$-ideal linked to $I$ with respect to the regular sequence $\underline{\alpha}=\alpha_{1}, \cdots, \alpha_{g}$. 
Then $\operatorname{dim}_{k}((\underline{\alpha}, m J) / m J) \geq g-r$.

Proof. Let $L_{1}(I) \subset R[X]$ be a generic link of $I$, then by Proposition 1.4, there exists $p \in \operatorname{Spec}(R[X])$ such that $\left(R[X]_{p}, L_{1}(I)_{p}\right)$ is a deformation of $(R, J)$. Set $(\tilde{R}, \tilde{J})=\left(R[X]_{p}, L_{1}(I)_{p}\right)$ and let $\underline{\alpha}=\tilde{\alpha}_{1}, \cdots, \tilde{\alpha}_{g}$ be the $\tilde{R}$ regular sequence defining the link $I \tilde{R} \sim \tilde{J}$. The $\tilde{R}$-ideal $I \tilde{R}$ has the same properties as $I, \nu(\tilde{J})=\nu(J)$, but since $I$ is generically a complete intersection, and $\tilde{J}$ is the localization of a first generic link of $I$ we also know that $\tilde{\alpha}_{1}, \cdots, \tilde{\alpha}_{g}$ generate $\tilde{J}$ generically $([3], 2.5)$. Moreover let $\tilde{m}$ be the maximal ideal of $\tilde{R}$, then

$$
\begin{aligned}
\operatorname{dim}_{k}((\underline{\tilde{\alpha}}, \tilde{m} \tilde{J}) / \tilde{m} J) & =\nu(J)-\nu(\tilde{J} /(\underline{\alpha})) \\
& =\nu(\tilde{J})-r(R / I R) \\
& =\nu(J)-r(R / I) \\
& =\nu(J)-\nu(J /(\underline{\alpha})) \\
& =\operatorname{dim}_{k}((\underline{\alpha}, m J) / m J) .
\end{aligned}
$$

Hence we do not change the assumptions or conclusions in the proposition if we replace $I, \underline{\alpha}, J$ by $I \tilde{R}, \underline{\alpha}, \tilde{J}$. However we may now assume that $J$ is generically generated by $\alpha_{1}, \cdots, \alpha_{g}$.

Now let $t=\operatorname{dim}_{k}((\underline{\alpha}, m J) / m J)$. After extending the residue class field if needed and changing $\alpha_{1}, \cdots, \alpha_{g}$ by elementary transformations, we may assume that $\alpha_{1}, \cdots, \alpha_{t}$ form part of a minimal generating set of $J$ and of $J_{p}$ for all $p \in \operatorname{Ass}(R / J)$. After factoring out $\alpha_{1}, \cdots, \alpha_{t}$ we are in the following situation: $(R, m)$ is a local Gorenstein ring, $I$ is a Cohen-Macaulay $R$-ideal, $r\left((R / I)_{p}\right) \leq r$ for all $p$ with $\operatorname{dim}(R / I)_{p} \leq(r+1)^{2}, J$ is linked to $I$ with respect to $\alpha, J$ is generically a complete intersection, but moreover $\underline{\alpha} \subset m J$, and grade $J=g-t$. We need to prove that grade $J \leq r$, since then $t \geq g-r$. From now on we write again grade $J=g$, and we will show $g \leq r$. We may assume $g>0$.

Let $\underline{f}=f_{1}, \cdots, f_{n}$ be a generating set of $J$. Since $\underline{\alpha} \subset m J$, there exists a $g \times n$ matrix $A$ with entries in $m$ such that $(\underline{\alpha})^{t}=A(\underline{f})^{t}$. Let $X$ be a generic $g \times n$ matrix, set $(\underline{\tilde{\alpha}})=X(\underline{f})^{t}$, consider the first generic link $L_{1}(J)$ $=L_{1}(\underline{f})=(\underline{\tilde{\alpha}}) R[X]: J R[X]$, and write $T=R[X]_{(m, X)}$. Because the entries of $A$ are in $m$, it follows from Proposition 1.4 that $\left(T, L_{1}(f) T\right)$ is a deformation of $(R, I)$. Since $R / I$ has the property that $r\left((R / I)_{p}\right) \leq r$ for all prime ideals $p$ with $\operatorname{dim}(R / I)_{p} \leq(r+1)^{2}$, any deformation of $R / I$, in particular $T / L_{1}(f) T$, has the same property (cf. [4], 2.3). But because the 
locus $\left\{p \mid p \in \operatorname{Spec}(R[X]), \quad r\left(R[X] / L_{1}(f)_{p}\right) \geq r+1\right\}=\{p \mid p \in \operatorname{Spec}(R[X])$, $\left.\nu\left(J R[X] /(\underline{\hat{\alpha}})_{p}\right) \geq r+1\right\}$ is defined by a homogeneous ideal in $R[X]$, it even follows that $r\left(\left(R[X] / L_{1}(f)\right)_{p}\right) \leq r$ for all $p \in \operatorname{Spec}\left(R[X] / L_{1}(f)\right)$ with $\operatorname{dim}\left(R[X] / L_{1}(f)\right)_{p} \leq(r+1)^{2}$.

For $q \in \operatorname{Ass}(R / J)$ let $\underline{h}=h_{1}, \cdots, h_{g}$ be a minimal generating set of $J_{q}$, let $Y$ be a generic $g \times g$ matrix, set $(\underline{\beta})^{t}=Y(\underline{h})^{t}$, and consider $L_{1}\left(J_{q}\right)=$ $L_{1}(\underline{h}) \subset R_{q}[Y]$. Then by [4], 2.13.b, $\left(R_{q}[Y], L_{1}(\underline{h})\right)$ is equivalent to the pair $\left(R_{q}[X], L_{1}(\underline{f}) R_{q}[X]\right)$, and hence also $R_{q}[Y] / L_{1}(\underline{h})$ has the property that $r\left(\left(R_{q}[Y] / L_{1}(\underline{h})\right)_{p}\right) \leq r$ for all prime ideals $p$ with $\operatorname{dim}\left(R_{q}[Y] / L_{1}(\underline{h})\right)_{p} \leq$ $(r+1)^{2}$. Instead of $J_{q}$ and $R_{q}$ we write again $J$ and $R$. We have to show that $g \leq r$.

Suppose that $g>r$. Then $p=\left(m, I_{g-r}(Y)\right) \in \operatorname{Spec}(R[Y])$, with $p \supset$ $(\underline{\beta}, \operatorname{det}(Y))=L_{1}(\underline{h})$, and $\operatorname{dim}\left(R[Y] / L_{1}(\underline{h})\right)_{p}=(r+1)^{2}$. However, $r(R[Y] /$ $\left.L_{1}(\underline{h})\right)_{p}=\nu\left((J R[Y] /(\underline{\beta}))_{p}\right)=r+1$, which is impossible by our assumptions. Therefore, $g \leq r$.

Proposition 2.2. Let $R$ be a Noetherian local ring which is a complete intersection, let $I$ be an unmixed $R$-ideal of height one, and assume that $I_{p}$ is principal for all $p \in \operatorname{Spec}(R)$ with $\operatorname{dim} R_{p} \leq 3$.

Then $I$ is a principal ideal.

Proof. By [2], Theorem 3.13, Exp. XI, any complete intersection of dimension at least 4 is parafactorial, i.e., the Picard group of its punctured spectrum is trivial.

Now assume $I$ is not principal and localize at a minimal prime $p$ such that $I_{p}$ is not princiral. Then $R_{p}$ is a complete intersection of dimension $\geq 4$ (by assumption) and $I_{p}$ represents an element in $\operatorname{Pic}(U)$ where $U=$ Spec $\left(R_{p}\right)-\left\{p_{p}\right\}$. Since $R_{p}$ is parafactorial this element is trivial. Hence there is an element of $a \in R$ such that $(a)_{q}=I_{q}$ for all $q_{p} \neq p_{p}$. This implies that $(a)_{p}: I_{p}$ is $p$-primary which is impossible or else $I_{p}=(a)_{p}$ since $I$ is unmixed.

TheORem 2.3. Let $R$ be a Noetherian local ring which is a complete intersection, let $I$ be a Cohen-Macaulay $R$-ideal of grade g, and assume that $(R, I)$ has a deformation $(\tilde{R}, \tilde{I})$ where $\tilde{I}$ is generically a complete intersection and $\tilde{R} / \tilde{I}$ satisfies $\left(G_{4}\right)$. Let $\underline{\alpha}=\alpha_{1}, \cdots, \alpha_{g} \subset I$ be a regular sequence with $(\underline{\alpha}) \neq I$, and set $J=(\underline{\alpha}): I$.

Then either $\underline{\alpha}$ form part of a minimal generating set of $J$, or both $I$ and $J$ are complete intersections. 
Proof. By [4], 2.16, there exists an $\tilde{R}$-ideal $\tilde{J}$ linked to $\tilde{I}$ with respect to a regular sequence $\underline{\alpha}$ such that $(\tilde{R}, \tilde{J})$ is a deformation of $(R, J)$. As in the proof of Proposition 2.1 one sees that $\underline{\alpha}$ is part of a minimal generating set of $J$ if and only if $\underline{\alpha}$ is part of a minimal generating set of $\tilde{J}$. Hence we may replace $I, \underline{\alpha}, J$, by $\tilde{I}, \underline{\alpha}, \tilde{J}$ and thus assume that $I$ is generically a complete intersection, and $R / I$ satisfies $\left(G_{4}\right)$.

Then we may apply Proposition 2.1 with $r=1$, and we obtain $\operatorname{dim}_{k}((\underline{\alpha}, m J) / m J) \geq g-1$. After extending the residue class field of $R$ if needed we may assume that $\alpha_{1}, \cdots, \alpha_{g-1}$ form part of a minimal generating set of $J$. Hence by factoring out $\left(\alpha_{1}, \cdots, \alpha_{g-1}\right)$ we do not change the assumptions and conclusion of the theorem (except possibly the assumption that $I$ is generically a complete intersection, which is irrelevant for the remainder of this proof).

Hence from now on $g=1$, and $\alpha_{1}=\alpha$. Let $m$ be the maximal ideal of $R$. Assuming that $\alpha \subset m J$ we will show that $J$ is principal. Then also $I$ is principal since $g=1$. Let $\underline{f}=f_{1}, \cdots, f_{n}$ be a generating set of $J$, then $\alpha=\sum_{i=1}^{n} C_{i} f_{i}$ with $C_{i} \in m$. For variables $X=X_{1}, \cdots, X_{n}$ set $\tilde{\alpha}=$ $\sum_{i=1}^{n} X_{i} f_{i} \in R[X]$ and consider the first generic link $L_{1}(J)=L_{1}(f)=\tilde{\alpha} R[X]$ : $J R[X]$. Since $C_{i} \in m,\left(R[X]_{(m, X)}, L_{1}(\underline{f}) R[X]_{(m, X)}\right)$ is a deformation of $(R, I)$, and it follows as in the proof of Proposition 2.1 that $R[X] / L_{1}(\underline{f})$ satisfies $\left(G_{4}\right)$.

Suppose that $J$ is not principal, then by Proposition 2.2 there exists a prime ideal $p \supset J$ with $\operatorname{dim} R_{p} \leq 3$ such that $J_{p}$ is not principal. On the other hand, $R / I$ being $\left(G_{4}\right)$ it follows that $I_{p}$ is either Gorenstein or the unit ideal, and hence $\nu\left(J_{p}\right) \leq g+1=2$. Thus $\nu\left(J_{p}\right)=2$, since $J_{p}$ is not principal. Moreover, any generic link of $J_{p}$ is equivalent (in the sense of Definition 1.1b) to a localization of a generic link of $J$, and hence also satisfies $\left(G_{4}\right)$. Therefore localizing at $p$ we may assume that $\operatorname{dim} R \leq 3$, and $\nu(J)=2$. Let $J=\left(h_{1}, h_{2}\right), \beta=Y_{1} h_{1}+Y_{2} h_{2} \in R\left[Y_{1}, Y_{2}\right]=S$, and $L_{1}(J)=$ $L_{1}\left(h_{1}, h_{2}\right)=\beta S: J S$. Then $\operatorname{dim} S / L_{1}(J) \leq 4$, and since $S / L_{1}(J)$ is $\left(G_{4}\right)$, it follows that $S / L_{1}(J)$ is Gorenstein. Therefore

$$
\nu\left((J S / \beta S)_{\left(m, Y_{1}, Y_{2}\right)}\right)=r\left(\left(S / L_{1}(J)\right)_{\left(m, Y_{1}, Y_{2}\right)}\right)=1
$$

which is impossible, since $\beta \in\left(Y_{1}, Y_{2}\right) J$ and therefore

$$
\nu\left((J S / \beta S)_{\left(m, Y_{1}, Y_{2}\right)}\right)=\nu(J)=2 .
$$




\section{§3. Applications}

The following corollary generalizes a result from [4] which states that if $I$ is an ideal in a regular local ring $R$ such that $I$ is in the linkage class of a complete intersection and $R / I$ satisfies $\left(G_{4}\right)$, then $I$ is Gorenstein.

Corollary 3.1. Let $R$ be a regular local ring, let $I$ be a perfect $R$ ideal which is generically a complete intersection, and assume that $R / I$ satisfies $\left(G_{4}\right)$.

Then for any $R$-ideal $J$ in the even linkage class of $I, r(R / J) \geq r(R / I)$. In particular if $I$ is in the even linkage class of a Gorenstein ideal, then $I$ is Gorenstein.

Proof. Assume that there is a sequence of links $I=I_{0} \sim I_{1} \sim \cdots \sim$ $I_{2 n}=J$. We will prove by induction on $n$ that $r(R / J) \geq r(R / I)$. Let $n=1$. We may suppose that $I$ is not a complete intersection. Let $\underline{\alpha}=$ $\alpha_{1}, \cdots, \alpha_{g}$ be the regular sequence defining the link $I \sim I_{1}$. By Theorem 2.3, $\underline{\alpha}$ is part of a minimal generating set of $I_{1}$, and hence $\nu\left(I_{1}\right)=\nu\left(I_{1} /(\underline{\alpha})\right)$ $+g=r(R / I)+g$. Let $\underline{\beta}=\beta_{1}, \cdots, \beta_{g}$ be the regular sequence giving the link $I_{1} \sim J$. Then $\nu\left(I_{1}\right) \leq \nu\left(I_{1} /(\underline{\beta})\right)+g=r(R / J)+g$. The above inequations now imply $r(R / J) \geq r(R / I)$. Now let $n \geq 2$. In [4], 2.17 we showed that in some local ring $S=R(X)$, which is obtained from $R$ by a purely transcendental extension of the residue class field, one can find a sequence of links $I S=J_{0} \sim J_{1} \sim \cdots \sim J_{2 n}$ such that $S / J_{2 n-2}$ is generically a complete intersection and satisfies $\left(G_{4}\right)$ (since $R / I$ has these properties), and moreover $r\left(S / J_{2 n}\right) \leq r(R / J)$. Then by induction hypothesis, applied to $I S$ and $J_{2 n-2}, \quad r(R / I)=r(S / I S) \leq r\left(S / J_{2 n-2}\right)$ and $r\left(S / J_{2 n-2}\right) \leq r\left(S / J_{2 n}\right)$. Combining the above inequalities we obtain $r(R / I) \leq r(R / J)$.

Let $R$ be a regular local ring with residue class field $k$, and let $I$ be an $R$-ideal. Consider the graded algebra $\Lambda_{\text {. }}=\operatorname{Tor}^{R}(R / I, k)$. We are interested in the condition $\Lambda_{1}^{2}=0$, which means that in a minimal free $R$ resolution of $R / I$, none of the Koszul relations on $I$ can occur among the minimal generators of the first syzygy module of $I$. It is well-known that $\Lambda_{1}^{2}=0$ if $I$ is a Gorenstein ideal of grade 3 , but not a complete intersection ([1]). The next corollary generalizes this result:

CoRollary 3.2. Let $R$ be a regular local ring, let $I$ be a perfect $R$ ideal of grade 3 , which is not a complete intersection, and assume that $I$ is generically a complete intersection and $R / I$ satisfies $\left(G_{4}\right)$.

Then $\Lambda_{1}^{2}=0$. 
Proof. Let $m$ be the maximal ideal of $R$, and let $F: 0 \rightarrow F_{3} \stackrel{\varphi_{3}}{\longrightarrow} F_{2}$ $\stackrel{\varphi_{2}}{\longrightarrow} F_{1} \stackrel{\varphi_{1}}{\longrightarrow} F_{0} \rightarrow R / I \rightarrow 0$ be a minimal free $R$-resolution of $R / I$. We choose bases $F_{2}=\oplus R d_{i}, F_{1}=\oplus \operatorname{Re}_{i}$, and set $f_{i}=\varphi_{1}\left(e_{i}\right)$.

Suppose that $\Lambda_{1}^{2} \neq 0$. Then we may assume that $\varphi_{2}\left(d_{1}\right)=f_{2} e_{1}-f_{1} e_{2}$. It is clear that ht $\left(f_{1} R+f_{2} R\right)=2$, since otherwise $f_{1}=a b_{1}$ and $f_{2}=a b_{2}$ with $0 \neq a \in m, b_{1} \in R, b_{2} \in R$, and hence $\varphi_{2}\left(d_{1}\right)=a\left(b_{2} e_{1}-b_{1} e_{2}\right)$ with $b_{2} e_{1}-$ $b_{1} e_{2} \in \operatorname{ker} \varphi_{1}$ which is a contradiction to the minimality of $F$. Because ht $\left(f_{1} R+f_{2} R\right)=2$, we may complete $f_{1}, f_{2}$ to a regular sequence $\underline{f}=f_{1}, f_{2}, f_{3}$ $\subset I$. Let $K .=K(\underline{f}, R)=\Lambda\left(R g_{1} \oplus R g_{2} \oplus R g_{3}\right)$ be the Koszul complex, and $u_{.}: K$. $\rightarrow F$. a morphism of complexes with $u_{0}=\mathrm{id}_{R}$. We may choose $u_{2}\left(g_{1} \wedge g_{2}\right)=-d_{1}$.

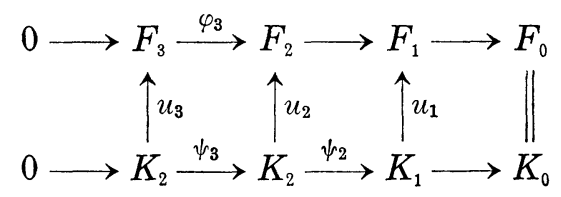

Set $J=(f): I$. Since the $R$-dual (denoted by -*) of the mapping cone of $u$. yields a resolution of $R / J$ ([6]), we obtain the following presentation of $J$ :

$$
K_{1}^{*} \oplus F_{2}^{*} \stackrel{\left(\begin{array}{cc}
\psi_{2}^{*} & 0 \\
u_{2}^{*} & \varphi_{3}^{*}
\end{array}\right)}{\longrightarrow} K_{2}^{*} \oplus F_{3}^{*} \longrightarrow J \longrightarrow 0
$$

Since $u_{2}\left(g_{1} \wedge g_{2}\right)=-d_{1}$ and hence $u_{2}^{*}\left(d_{1}^{*}\right) \notin m K_{2}^{*}$, it follows that $\nu(J)<$ $\operatorname{rank}\left(K_{2}^{*} \oplus F_{3}^{*}\right)=3+r(R / I)$. Thus $f_{1}, f_{2}, f_{3}$ cannot be part of a minimal generating set of $J$. This is a contradiction to Theorem 2.3.

\section{REFERENCES}

[1] D. Buchsbaum and D. Eisenbud, Algebra structures for finite free resolutions, and some structure theorems for ideals of codimension 3, Amer. J. Math., 99 (1977), 447-485.

[2] A. Grothendieck, Cohomologie locale des faisceaux cohérents et Théorèmes de Lefschetz locaux et globaux (SGA II), Exposé XIV, Advanced Studies in Mathematics, Vol. II (1968), North Holland Publishing Co., Amsterdam.

[ 3 ] C. Huneke and B. Ulrich, Divisor class groups and deformations, Amer. J. Math., 107 (1985), 1265-1303.

[ 4 ] — - The structure of linkage, Ann. of Math., 126 (1987), 277-334.

[5] —-, Algebraic linkage, to appear, Duke J. Math. 
[6] C. Peskine and L. Szpiro, Liaison des variétés algébriques, Invent. Math., 26 (1974), 271-302.

C. Huneke

Department of Mathematics

Purdue University

West Lafayette, IN 47907

B. Ulrich

Department of Mathematics

Michigan State University

East Lansing, MI 48824 